\title{
Pain Is Inevitable But Suffering Is Optional: Relationship of Pain Coping Strategies to Performance in Multistage Ultramarathon Runners
}

\author{
Kevin N. Alschuler, $\mathrm{PhD}^{1,2}$; Brian J. Krabak, $\mathrm{MD}^{1,3}$; Anna L. Kratz, $\mathrm{PhD}^{4}$; Mark P. Jensen, $\mathrm{PhD}^{1}$; \\ Dave Pomeranz, MD ${ }^{5}$; Patrick Burns, $\mathrm{MD}^{6}$; Joshua Bautz, $\mathrm{MD}^{7}$; Claire Nordeen, $\mathrm{MD}^{8}$; \\ Crystal Irwin, $\mathrm{DO}^{9}$; Grant S. Lipman, $\mathrm{MD}^{6}$ \\ ${ }^{1}$ Department of Rehabilitation Medicine, University of Washington, Seattle, WA; ${ }^{2}$ Department of Neurology, University of Washington, Seattle, WA; \\ ${ }^{3}$ Department of Orthopedics and Sports Medicine, University of Washington, Seattle, WA; ${ }^{4}$ Department of Physical Medicine and Rehabilitation, Univer- \\ sity of Michigan, Ann Arbor, MI; ${ }^{5}$ Northern Inyo Hospital, Bishop, CA; ${ }^{6}$ Department of Emergency Medicine, Stanford University School of Medicine, \\ Stanford, CA; ${ }^{7}$ Department of Emergency Medicine, Naval Medical Center Camp Lejeune, Camp Lejeune, NC; ${ }^{8}$ Department of Emergency Medicine, \\ University of Washington, Seattle, WA; ${ }^{9}$ LA County - University Southern California Emergency Medicine Residency, Keck School of Medicine, Los \\ Angeles, $C A$
}

\begin{abstract}
Introduction-Ultramarathon runners commonly endure musculoskeletal pain during endurance events. However, the effect of pain coping skills on performance has not been examined.

Methods-A prospective observational study during three $250 \mathrm{~km}$ (155 mi), 6 stage ultramarathons was conducted. Finish line surveys were completed after each of the four $40 \mathrm{~km}(25 \mathrm{mi})$ and one $80 \mathrm{~km}(50 \mathrm{mi})$ stages of racing. Variables gathered included pain intensity, pain coping strategy use, pain interference, finishing position (quintile), and successful race completion.

Results-A total of 204 participants (age $41.4 \pm 10.3$ y; $73 \%$ male) reported average pain intensity of 3.9 $( \pm 2.0 \mathrm{SD})$ and worst pain intensity of $5.3( \pm 2.3)$ on a 0 to 10 scale. They used greater adaptive pain coping strategies $(3.0 \pm 1.3)$ relative to maladaptive strategies $(1.3 \pm 1.1)$. Worst pain and pain interference increased over each stage of the race for all runners $(P<0.001)$, with worst pain being significantly different by finishing status $(P=0.02)$. Although all runners endured increased pain and interference, the nonfinishers $(28$ [14\%]) had significantly greater differences in changes in pain intensity $(P<0.01)$ and pain interference $(P<0.001)$. Maladaptive pain coping strategies were more common in nonfinishers; with each 1-point increase ( $0-6$ scale), there was a 3 times increase in odds of not finishing the race.

Conclusions-Although increased pain intensity and pain interference was found in all multistage ultramarathon runners, successful event completion was significantly associated with less maladaptive pain coping. Training in coping with pain may be a beneficial part of ultramarathon preparation.
\end{abstract}

Keywords: endurance, ultramarathon, running, musculoskeletal pain, pain coping skills

\section{Introduction}

Ultramarathon running events are single-stage or multistage foot races that are longer than the standard $42.2 \mathrm{~km}(26.2$ mi) marathon. In addition to the long distances of these

Corresponding author: Kevin N. Alschuler, PhD, University of Washington, Box 358815, 1536 N 115th St, Seattle, Washington 98133; e-mail:kalschul@uw.edu.

Submitted for publication May 2019.

Accepted for publication October 2019. events, they often take place in desert or mountainous terrain that increases the potential for injuries and illnesses associated with prolonged exposure and exertion in wilderness conditions. ${ }^{1}$ Given the prolonged exertion in extreme condition, it is not surprising that pain and injury are common among ultramarathon runners. Up to $52 \%$ of ultramarathon runners report alterations in training, ${ }^{2}$ and $65 \%$ report lost training days due to some form of musculoskeletal (MSK) pain. ${ }^{3}$ The source of MSK pain can range from mild muscle soreness and cramps secondary to prolonged exercise to more severe pain from a specific injury. ${ }^{4,5}$ 
The annual incidence of MSK injuries in ultramarathon runners are similar to shorter distance runners, ${ }^{6-8}$ with the most common training injuries involving the knees and iliotibial band. Similar to training, MSK pain is experienced by as many as 22 to $24 \%$ of ultramarathon runners during and after a race, with the knees and Achilles tendons most commonly affected. ${ }^{9,10}$ Although MSK pain appears to be almost inevitable in endurance running, little is known about how ultramarathon athletes use pain coping mechanisms to minimize suffering while optimizing performance.

Although it is reasonable to assume pain could pose a barrier to optimal performance, this issue remains largely unexamined. Exercise has been shown to have an analgesic effect in both animals ${ }^{11}$ and humans, ${ }^{12,13}$ though the mechanism is unclear and thought to be a complex interplay among signaling pathways, cognition, and perception. Other factors such as sex,${ }^{14}$ competition, ${ }^{15}$ and age ${ }^{16}$ have been shown to be associated with pain perception during exercise. Theories of pain modulation create a construct for further understanding of pain signaling. ${ }^{17}$ The gate control theory has facilitated an understanding of how nociceptive signals from the periphery can dampen or block afferent pain signals. Studies show that even at rest, athletes have demonstrated a higher pain tolerance than their nonactive peers, suggesting the presence of more effective inhibitory processes in these individuals. ${ }^{18}$ This phenomenon does not appear to be a fixed trait; the degree of the pain tolerance changes throughout an athlete's training cycles ${ }^{19}$ and further increases after an athlete exercises. ${ }^{15,20}$ Moreover, research also refutes the notion that athletes feel less pain; the threshold levels at which they report noxious stimuli as painful is unchanged from their nonactive peers. ${ }^{18}$ However, athletes do demonstrate increased ability to discriminate among noxious stimuli. ${ }^{21}$

Most pain research in athletes has focused around "stoicism," which suggests decreased emotional reactivity to pain, decreased fear of pain, and increased confidence in the ability to handle pain. ${ }^{22,23} \mathrm{We}$ do not know how pain intersects with athletic performance and, specifically, whether the way individuals respond to pain can affect the pain-performance intersection. Research from nonathlete populations provides a foundation for examining this relationship, suggesting that an individual's cognitive (beliefs) and behavioral (coping) responses influence that individual's function in the presence of pain; responses classified as "adaptive" are hypothesized to improve function, and responses classified as "maladaptive" are hypothesized to interfere with function when pain is present. ${ }^{24,25}$

The objective of this research was to explore the relationship that ultramarathon runners' pain experience and coping mechanisms have with their race performance. Specifically, we sought to understand 1) the relationship between trends in pain intensity, pain coping strategies, and pain interference and the performance outcomes of finishing position (by quintile) and race completion and 2) whether specific adaptive or maladaptive coping strategies were associated with these outcomes. We hypothesized that higher levels of performance (finishing vs not finishing, or higher finishing position) would be associated with greater use of adaptive pain coping strategies and lesser use of maladaptive coping strategies.

\section{Methods \\ RECRUITMENT AND PROCEDURES}

Data were collected as part of a study on pain coping strategies in ultramarathon runners. ${ }^{26}$ Participants were recruited from registrants at the 2016 RacingThePlanet $250 \mathrm{~km}$ (155 mi) 6-stage ultramarathon races in the Atacama Desert in Chile, Gobi Desert in China, and Namibian Desert. The races had 4 initial stages of $40 \mathrm{~km}(25 \mathrm{mi})$ each, with an $80 \mathrm{~km}(50 \mathrm{mi})$ fifth stage, followed by a final $8 \mathrm{~km} \mathrm{(5} \mathrm{mi)}$ sixth stage. The races require the runners to carry their own food and gear for the duration of the event, with the race organizers supplying water and sleeping tents. Following precedent established in prior research, for analytic purposes we collapsed participant data across the 3 races into a single cohort owing to the similar logistical demands and history of similar participant characteristics. ${ }^{1,27-29}$

All race entrants were offered the opportunity to participate in the study at the time of mandatory prerace check-in. Those who met inclusion criteria (age $\geq 18 \mathrm{y}$, able to read and write in English to be able to answer study questionnaires) signed an informed consent form and completed a demographics questionnaire. They then completed a daily questionnaire at the finish line after completion of each day's stage for stages 1 through 5. (Given the brevity of the sixth stage and resulting inconsistency with the physical demand of the first 5 stages, data were not collected after that stage.) Participation was voluntary, and no compensation was provided. Ethical approval was provided by the University of Washington's human subjects division.

\section{MEASURES}

\section{Demographics and daily questionnaire}

Study participants provided data on their age, sex, and running experience (marathons entered and completed and ultramarathons entered and completed). After each stage of racing participants completed a daily questionnaire (using the anchor, "In today's stage...") that was constructed with primarily single-item questions assessing important constructs to maximize brevity and thus minimize participant burden. The use of 1- and 2-item scales 
derived from longer measures is supported in the literature. $^{30}$

\section{Pain intensity}

Participants were asked to rate their average and worst pain intensity during the previous stage using the 0 to 10 numerical rating scale, a well-validated measure of pain intensity that is commonly used in pain research. ${ }^{31}$

\section{Race completion and finish position}

Race completion was assigned a dichotomous outcome (finisher vs nonfinisher), with accuracy ensured through race organizer official records. The participant's finish position was defined in quintiles: top $10 \%, 11$ to $25 \%, 26$ to $50 \%, 51$ to $75 \%$, and $>75 \% .^{32}$

\section{Exertion}

Participants provided a rating of perceived exertion using the well-validated Borg scale. ${ }^{33}$ The rating of perceived exertion is reported on a scale of 6 (no exertion at all) to 20 (maximal exertion).

\section{Coping}

Three categories of coping were assessed: experiential awareness, adaptive pain coping, and maladaptive pain coping. Items were selected or adapted from a number of validated questionnaires ${ }^{30,34,35}$ and were uniformly answered on a scale from 0 (never) to 6 (always).

Experiential awareness. Participants indicated the extent to which they 1) felt optimistic, 2) felt an urge to keep going, 3) maintained a sense of mindful observation, and 4) maintained a sense of automaticity.

Adaptive pain-related coping. Questions believed to be associated with adaptive pain coping included the extent to which participants 1) ignored their pain, 2) saw pain as a challenge, and 3) tried not to let pain bother them.

Maladaptive pain-related coping. Questions believed to be associated with maladaptive pain coping included the extent to which participants 1) felt they could not stand their pain anymore, 2) felt defeated by their pain, 3) felt frightened by their pain, and 4) felt an urge to stop because of the pain.

For each of the coping domains, the items were averaged to generate a composite score, and all demonstrated good internal consistency (Cronbach's alpha for experiential awareness $=0.83$; adaptive pain coping $=0.78$; and maladaptive pain coping $=0.89)$. $^{26}$

\section{STATISTICAL ANALYSIS}

Univariate descriptive statistics were computed with the demographic, pain, and coping variables to describe the sample and key study measures. Prior to conducting primary analyses, stage-level variables were aggregated (averaged across the stages) for inclusion in models examining between-person associations. To examine differences in changes in pain intensity (using worst pain, considering its larger effect on the study outcome relative to average pain) and pain interference over race stages by finish status, 2 mixed effects multilevel models were conducted-one for race completion and one for finish status. Use of multilevel models has a number of advantages, including the ability to model within-person and between-person variance simultaneously, handling of autocorrelation of within-person observations, and retention of individuals with some missing within-person data. Our first step was to test whether these variables changed across stage; the linear and curvilinear (quadratic) effects of time were examined. Once the general shape (linear or curvilinear) of the change over time was determined, interaction terms (eg, the interaction of race stage with finish status) were created to test for differences in the trajectories of pain and pain interference over time by finish status.

Variations of general linear models with the 3 coping variables (adaptive general, adaptive pain, maladaptive pain) included as simultaneous dependent variables were used to predict whether the racer finished (in a binary logistic regression) and their finishing quintile (in an ordinal regression). All underlying statistical assumptions were tested before regression models were conducted. In both cases, there was no evidence of multicollinearity (all variance inflation factor values $<2.86$ ). For the binary logistic regression, linearity of the continuous variables with respect to the logit of the dependent variable was assessed via the Box-Tidwell (1962) procedure. A Bonferroni correction was applied using all 12 terms in the model, resulting in statistical significance being accepted at $P<0.004$. Based on this assessment, all continuous independent variables were found to be linearly related to the logit of the dependent variable. For the ordinal regression, the assumption of proportional odds was met, as assessed by a full likelihood ratio test comparing the fit of the proportional odds model to a model with varying location parameters $\left(\chi^{2}(18)=14.71, P=0.682\right)$. Statistical tests were performed using SAS version 9.4 (SAS Institute, Cary, NC).

\section{Results}

There was a total of 427 entrants in the three 2016 races, with 204 (48\%) enrolled in the study. Demographics of the participants are described in Table 1. Participants were 
Table 1. Participant demographics and finish rate statistics by race

\begin{tabular}{lccc}
\hline Variable (possible range) & $\begin{array}{c}\text { Full Sample }(n=204) \\
\text { Mean } \pm \text { SD or } \%\end{array}$ & $\begin{array}{c}\text { Finishers }(n=176) \\
\text { Mean } \pm \text { SD or } \%\end{array}$ & $\begin{array}{c}\text { Nonfinishers }(n=28) \\
\text { Mean } \pm \text { SD or } \%\end{array}$ \\
\hline Age (y) & $41 \pm 10$ & $41 \pm 10$ & $46 \pm 11$ \\
Sex (male) & $73 \%$ & $73 \%$ & $70 \%$ \\
Previous ultramarathon experience & & & $11 \pm 18$ \\
$\quad$ Attempted & $5 \pm 8$ & $9 \pm 15$ & $11 \pm 18$ \\
Completed & $5 \pm 7$ & $9 \pm 15$ & $91 \pm 24$ \\
Completed $(\%)$ & $90 \pm 24$ & $99 \pm 10$ & $14 \%$ \\
Race & & & $11 \%$ \\
Namibia & & $86 \%$ & $14 \%$ \\
Gobi & & $89 \%$ & $86 \%$ \\
$\quad$ Atacama & & & \\
\hline
\end{tabular}

middle aged, with the majority being men. Finishers differed from nonfinishers in terms of age (nonfinishers $>$ finishers), but they were otherwise similar. There was no statistically significant difference in finishing rate among races. Descriptive data are provided in Table 2. Over the course of the race, the average pain intensity and pain interference were in the low to mild ranges. The participants endorsed using more adaptive coping strategies compared with maladaptive coping strategies. Coping trajectories by finish status are shown in Figure 1.

Table 2. Descriptive characteristics for primary study variables

\begin{tabular}{|c|c|}
\hline Variable (possible range) & Mean \pm SD or $n(\%)$ \\
\hline Percent time thinking of pain $(0-100)^{\mathrm{a}}$ & $30.9 \pm 21.0$ \\
\hline Average pain intensity $(0-10)^{\mathrm{a}}$ & $3.9 \pm 2.0$ \\
\hline Worst pain intensity $(0-10)^{\mathrm{a}}$ & $5.2 \pm 2.0$ \\
\hline Pain interference $(0-10)^{\mathrm{a}}$ & $4.1 \pm 2.0$ \\
\hline Experiential awareness $(0-6)^{\mathrm{a}}$ & $3.7 \pm 1.0$ \\
\hline Adaptive pain coping $(0-6)^{\mathrm{a}}$ & $3.0 \pm 1.0$ \\
\hline Maladaptive pain coping $(0-6)^{\mathrm{a}}$ & $1.3 \pm 1.0$ \\
\hline \multicolumn{2}{|l|}{ No. of stages finished (out of 5) } \\
\hline 1 & $7(3)$ \\
\hline 2 & $5(3)$ \\
\hline 3 & $14(7)$ \\
\hline 4 & $2(1)$ \\
\hline 5 & $176(86)$ \\
\hline \multicolumn{2}{|l|}{ Finished race } \\
\hline Yes & $176(86)$ \\
\hline No & $28(14)$ \\
\hline \multicolumn{2}{|l|}{ Finishing quintile } \\
\hline Top $10 \%$ & $24(12)$ \\
\hline$>10 \%-25 \%$ & $35(17)$ \\
\hline$>25 \%-50 \%$ & $41(20)$ \\
\hline$>50 \%-75 \%$ & $48(24)$ \\
\hline$>75 \%$ & $28(14)$ \\
\hline Did not finish & $28(14)$ \\
\hline
\end{tabular}

${ }^{a}$ Averaged across the assessments obtained from the participants from each race stage.
Worst pain intensity and pain interference demonstrated a statistically significant linear worsening over the stages (worst pain $P<0.001$; pain interference $P<0.001)$. There were no significant curvilinear effects of stage (time) on either worst pain intensity or pain interference. Change in worst pain intensity over the stages was significantly different by finish status $(P<0.02)$. Observation of simple slope values for finishers $(P<0.001)$ versus nonfinishers $(P<0.01)$ indicated that although worst pain intensity increased with each stage for both finishers and nonfinishers, the increase in pain intensity was greater for those who did not finish (Figure 2). Similarly, change in pain interference over the stage was significantly different by finish status $(P<0.01)$. Although pain interference increased with each stage for both finishers and nonfinishers, the increase was greatest for those who did not finish the race $(P<0.001)$ (Figure 3$)$.

Logistic regression examining the effects of age, worst pain, exertion, adaptive general coping, maladaptive pain coping, and adaptive pain coping on finishing status was significant $(P<0.001)$ and accounted for $33 \%$ of the variance in whether a person finished. The model correctly classified 182 cases $(89 \%)$. Of these 6 predictor variables, only 2 were statistically significant: age and maladaptive pain coping (Table 3). Older age was associated with lower odds of finishing, such that with each additional year of age, there was an approximately $10 \%$ reduction in the odds of finishing the race. For each 1-point increase in the maladaptive pain coping measure, there was a $68 \%$ reduction in the odds of finishing the race.

The results of an ordinal logistic regression with proportional odds examining the effects of age, worst pain, exertion, adaptive general coping, maladaptive pain coping, and adaptive pain coping on finishing quintile showed that none of the predictors were related to odds of finishing in a higher or lower quintile $(P>0.2)$. 

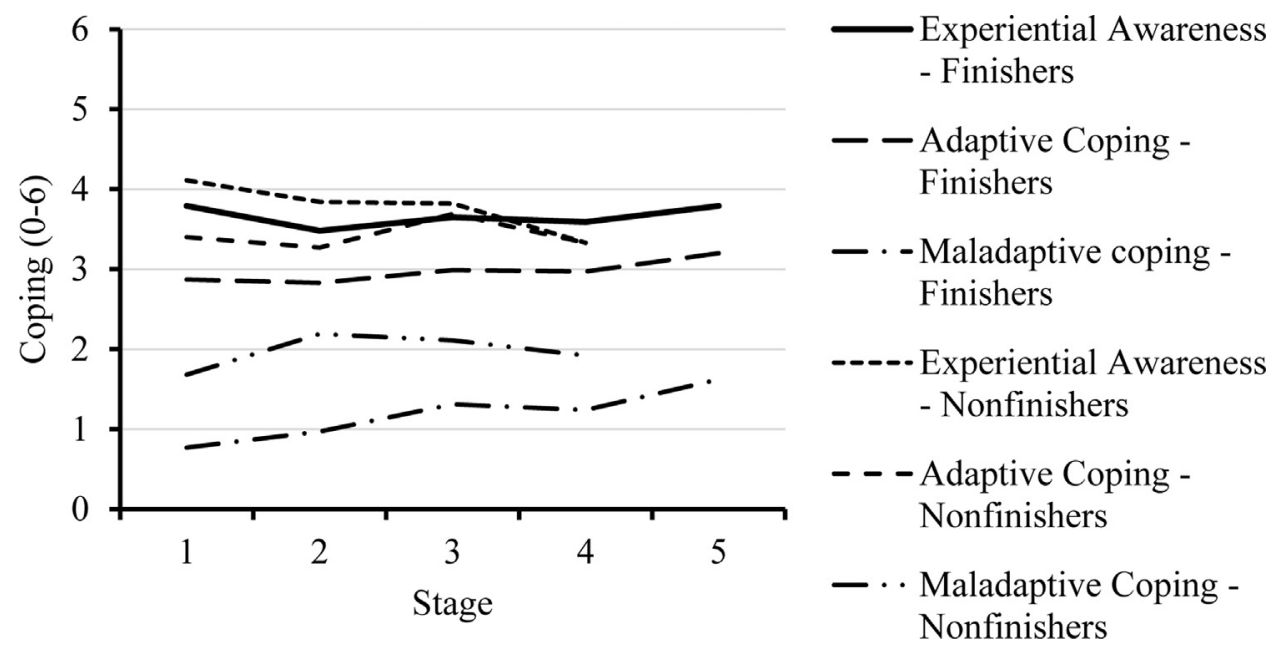

Figure 1. Trajectories of coping by finish status. Lines depict group means at each race stage.

\section{Discussion}

The study hypothesis on the relationship between pain coping and performance in multistage ultramarathon runners was partially supported: a coping profile that emphasized lesser use of maladaptive coping strategies was associated with a greater likelihood of race completion. However, the extent to which a study participant used adaptive coping strategies was not associated with race completion. Additionally, neither adaptive nor maladaptive coping strategy use was associated with better performance, as defined by a higher quintile finish position. Increased pain tolerance has been seen in diverse groups of athletes including runners, ${ }^{36}$ rowers ${ }^{37}$ fighters, ${ }^{38}$ swimmers, ${ }^{19}$ and even elite dancers, ${ }^{39}$ and it is possible that those who did not "suffer" as much (ie, used fewer maladaptive coping strategies) were able to better tolerate the inevitable pain of running $250 \mathrm{~km}(155 \mathrm{mi})$ and successfully complete the race.

We found that moderate average pain intensity was common among multistage ultramarathon runners. Repeated assessment of runners showed a linear worsening of worst pain intensity (ie, pain intensity increased as the runner progressed from stage to stage), with a more rapid worsening among those who did not finish the event. Conversely, there was no curvilinear relationship, meaning that there was no phenomenon wherein pain peaked in the middle of the race but then lessened as the finish line approached. Taken together, this supports the possibility that pain is present at sufficient levels in these races to represent an independent risk factor for not finishing an event.

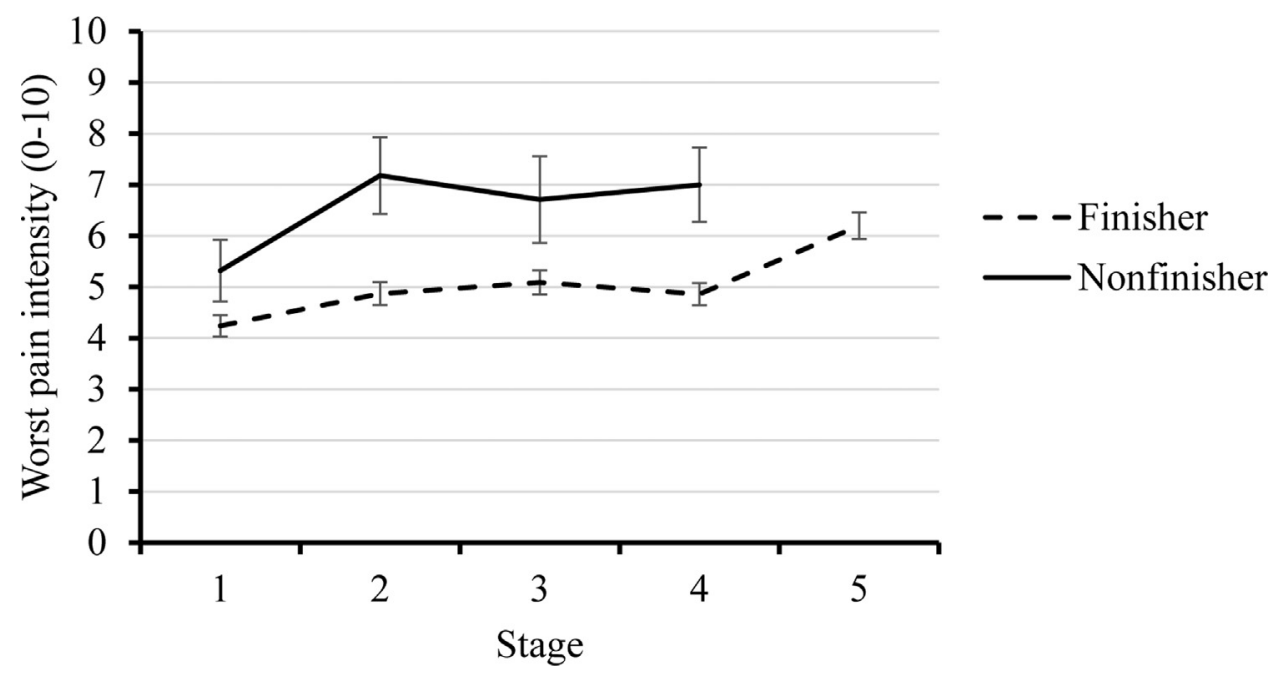

Figure 2. Trajectories of worst pain intensity by finish status. Lines depict group means at each race stage with standard error bars. 


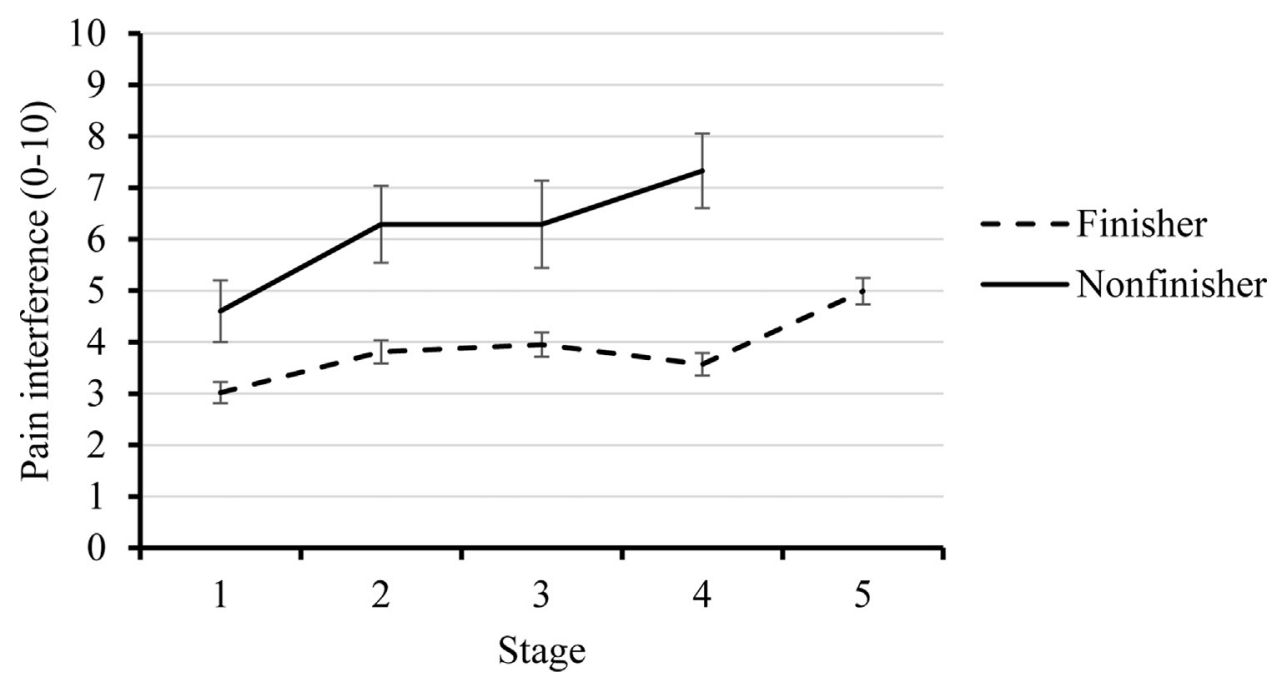

Figure 3. Trajectories of pain interference by finish status. Lines depict group means at each race stage with standard error bars.

Given the orientation of this population toward voluntary participation in a uniquely challenging physical task, it is not surprising that these individuals used adaptive coping strategies more frequently and maladaptive coping strategies less frequently, despite the presence of moderate levels of pain. This coping profile differs from pain populations evaluated in clinical settings, especially chronic pain, where there is a greater propensity for maladaptive coping strategy use relative to adaptive coping strategies. ${ }^{24}$

There was a substantial decrease in performance (signified by an increase in the likelihood of dropping out of the race) when use of maladaptive coping increased. This is consistent with the literature on other pain populations (eg, patients with nonathletic chronic pain) that suggests a substantial role for maladaptive coping in objective pain outcomes. ${ }^{40}$ Additionally, there was a small increase in the likelihood of dropping out of the race for every $10 \mathrm{y}$ of age, which notably differs from prior research showing that the risk of injury decreases with age. ${ }^{9}$ Similar findings did

Table 3. Logistic regression results predicting finish status from general adaptive, pain-specific adaptive, and pain-specific maladaptive coping strategies, controlling for age, average worst pain, and average Borg rating ( $\mathrm{df}$ for each parameter $=1$ )

\begin{tabular}{lccc}
\hline Predictor & P value & Odds ratio & $95 \%$ CI \\
\hline Age $(\mathrm{y})$ & $<0.001$ & 0.90 & $0.9-1.0$ \\
Worst pain intensity & 0.9 & 1.02 & $0.7-1.4$ \\
Borg & 0.3 & 0.90 & $0.7-1.1$ \\
Experiential awareness & 0.5 & 1.25 & $0.7-2.4$ \\
Adaptive pain coping & 0.2 & 0.72 & $0.4-1.2$ \\
Maladaptive pain coping & $<0.001$ & 0.32 & $0.2-0.6$ \\
Constant & $<0.001$ & 2.25 & \\
\hline
\end{tabular}

not emerge for either age or maladaptive coping for our other performance outcome of finish position. Although it may be the case that neither age nor maladaptive coping predict running speed, we suspect that the finding in this case may be due to 2 factors: 1) that a vast majority of participants prioritize finishing over speed and 2) the heterogeneity of the population in terms of age, sex, experience, physical ability, and more. In the absence of sufficient data (and sample size) to control for all of these variables, any effect of pain coping on speed-based outcomes is washed out.

There are limitations to this study. Although the present study illustrates differences between the finisher and nonfinisher groups in terms of how they coped with pain, our understanding of nonfinishers is incomplete. We did not collect data from nonfinishers at the point that they withdrew, so we do not have an understanding of the extent to which their withdrawal relates to the pain they were coping with throughout the study relative to other factors. For the variables we do have, analysis is limited by statistical power because the number of nonfinishers $(n=28)$ is relatively small. In an effort to minimize participant burden and maximize study participation, we conducted a brief data collection at each timepoint that focused exclusively on the participants' pain experience (eg, intensity, interference, and coping) and did not consider other variables across the biospyschosocial spectrum that may also contribute to finish position or race completion, such as body mass index, degree of social support for race participation and completion, and trait psychological function (eg, trait optimism or anxiety level). This includes not gathering injury data or querying the cause of the pain, which could have allowed a root cause analysis of the pain experience. In a similar spirit, we also opted to use brief measures of pain coping. This approach is justified in the literature ${ }^{30}$ 
but does raise the possibility of lower reliability, which can limit power, reduce the estimates of the associations among variables, and have less content validity than their parent scales. Finally, we recognize that the study was conducted on the unique subpopulation of individuals who participate in multistage ultramarathons, and results may not be generalizable to other populations or athletes in other endurance activities.

\section{Conclusions}

Worst pain intensity and pain interference was found to increase over the course of in multistage ultramarathon events. The observed strong direct relationship of decreased use of maladaptive coping and successful completion of a multistage ultramarathon suggests there may be value in exploring proactive approaches to train athletes in the utilization of effective pain coping strategies.

Author Contributions: Study concept and design (KNA, GSL, ALK, MPJ, BJK); obtaining funding (KNA, GSL, BJK); acquisition of the data (DP, PB, JB, CN, CI); analysis of the data (KNA, GSL, ALK, MPJ, BJK); drafting of the manuscript (KNA, GSL, ALK, MPJ, DP, PB, JB, $\mathrm{CN}, \mathrm{CI}, \mathrm{BJK}$ ); critical revision of the manuscript (KNA, GSL, ALK, MPJ, DP, PB, JB, CN, CI, BJK); approval of final manuscript (KNA, GSL, ALK, MPJ, DP, PB, JB, CN, CI, BJK).

Financial/Material Support: The authors wish to thank RacingThePlanet for their financial support of this study.

Disclosures: None.

\section{References}

1. Krabak BJ, Lipman GS, Waite BL, Rundell SD. Exerciseassociated hyponatremia, hypernatremia, and hydration status in multistage ultramarathons. Wilderness Environ Med. 2017;28(4):291-8.

2. Hoffman MD, Fogard K. Factors related to successful completion of a 161-km ultramarathon. Int J Sports Physiol Perform. 2011;6(1):25-37.

3. Hoffman MD, Krishnan E. Health and exercise-related medical issues among 1,212 ultramarathon runners: baseline findings from the Ultrarunners Longitudinal TRAcking (ULTRA) Study. PLoS One. 2014;9(1), e83867.

4. Krabak BJ, Waite B, Lipman G. Injury and illnesses prevention for ultramarathoners. Curr Sports Med Rep. 2013;12(3): 183-9.

5. Krabak BJ, Waite B, Lipman G. Evaluation and treatment of injury and illness in the ultramarathon athlete. Phys Med Rehabil Clin N Am. 2014;25(4):845-63.

6. Hoffman MD. Injuries and health considerations in ultramarathon runners. Phys Med Rehabil Clin $N$ Am. 2016;27(1):203-16.

7. Teixeira RN, Lunardi A, da Silva RA, Lopes AD, Carvalho CR. Prevalence of musculoskeletal pain in marathon runners who compete at the elite level. Int J Sports Phys Ther. 2016;11(1):126-31.
8. van Gent RN, Siem D, van Middelkoop M, van Os AG, Bierma-Zeinstra SM, Koes BW. Incidence and determinants of lower extremity running injuries in long distance runners: a systematic review. Br J Sports Med. 2007;41(8):469-80.

9. Krabak BJ, Waite B, Schiff MA. Study of injury and illness rates in multiday ultramarathon runners. Med Sci Sports Exerc. 2011;43(12):2314-20.

10. Hoffman MD, Pasternak A, Rogers IR, Khodaee M, Hill JC, Townes DA, et al. Medical services at ultra-endurance foot races in remote environments: medical issues and consensus guidelines. Sports Med. 2014;44(8):1055-69.

11. Kuphal KE, Fibuch EE, Taylor BK. Extended swimming exercise reduces inflammatory and peripheral neuropathic pain in rodents. J Pain. 2007;8(12):989-97.

12. Koltyn KF. Analgesia following exercise: a review. Sports Med. 2000;29(2):85-98.

13. Hoffman MD, Lee J, Zhao H, Tsodikov A. Pain perception after running a 100-mile ultramarathon. Arch Phys Med Rehabil. 2007;88(8): 1042-8.

14. Sternberg WF, Bokat C, Kass L, Alboyadjian A, Gracely RH. Sex-dependent components of the analgesia produced by athletic competition. J Pain. 2001;2(1):65-74.

15. Sternberg WF, Bailin D, Grant M, Gracely RH. Competition alters the perception of noxious stimuli in male and female athletes. Pain. 1998;76(1-2):231-8.

16. Naugle KM, Naugle KE, Riley 3rd JL. Reduced modulation of pain in older adults after isometric and aerobic exercise. J Pain. 2016;17(6):719-28.

17. Ropero Pelaez FJ, Taniguchi S. The Gate Theory of Pain revisited: modeling different pain conditions with a parsimonious neurocomputational model. Neural Plast. 2016;2016: 4131395.

18. Tesarz J, Schuster AK, Hartmann M, Gerhardt A, Eich W. Pain perception in athletes compared to normally active controls: a systematic review with meta-analysis. Pain. 2012;153(6):1253-62.

19. Scott V, Gijsbers K. Pain perception in competitive swimmers. Br Med J (Clin Res Ed). 1981;283(6284):91-3.

20. Guieu R, Blin O, Pouget J, Serratrice G. Nociceptive threshold and physical activity. Can J Neurol Sci. 1992;19(1): 69-71.

21. Janal MN, Glusman M, Kuhl JP, Clark WC. Are runners stoical? An examination of pain sensitivity in habitual runners and normally active controls. Pain. 1994;58(1):109-16.

22. Geva N, Defrin R. Enhanced pain modulation among triathletes: a possible explanation for their exceptional capabilities. Pain. 2013;154(11):2317-23.

23. Johnson MH, Stewart J, Humphries SA, Chamove AS. Marathon runners' reaction to potassium iontophoretic experimental pain: pain tolerance, pain threshold, coping and self-efficacy. Eur J Pain. 2012;16(5):767-74.

24. Tan G, Teo I, Anderson KO, Jensen MP. Adaptive versus maladaptive coping and beliefs and their relation to chronic pain adjustment. Clin J Pain. 2011;27(9):769-74.

25. Geisser ME, Robinson ME, Riley JL. Pain beliefs, coping, and adjustment to chronic pain - let's focus more on the negative. Pain Forum. 1999;8(4):161-8. 
26. Alschuler KN, Kratz AL, Lipman GS, Krabak BJ, Pomeranz D, Burns P, et al. How variability in pain and pain coping relates to pain interference during multi-stage ultramarathons. Pain. 2019;160(1):257-62.

27. Lipman GS, Shea K, Christensen M, Phillips C, Burns P, Higbee R, et al. Ibuprofen versus placebo effect on acute kidney injury in ultramarathons: a randomised controlled trial. Emerg Med J. 2017;34(10):637-42.

28. Lipman GS, Sharp LJ, Christensen M, Phillips C, DiTullio A, Dalton A, et al. Paper tape prevents foot blisters: a randomized PREvention Trial Assessing Paper tape in Endurance Distances II (Pre-TAPED II). Clin J Sport Med. 2016;26(5): 362-8.

29. Hoffman MD, Hew-Butler T, Stuempfle KJ. Exercise-associated hyponatremia and hydration status in 161-km ultramarathoners. Med Sci Sports Exerc. 2013;45(4):784-91.

30. Jensen MP, Keefe FJ, Lefebvre JC, Romano JM, Turner JA. One- and two-item measures of pain beliefs and coping strategies. Pain. 2003;104(3):453-69.

31. Jensen MP, Turner JA, Romano JM, Fisher LD. Comparative reliability and validity of chronic pain intensity measures. Pain. 1999;83(2):157-62.

32. Lipman GS, Krabak BJ, Rundell SD, Shea KM, Badowski N, Little C. Incidence and prevalence of acute kidney injury during multistage ultramarathons. Clin J Sport Med. 2016;26(4): 314-9.

33. Borg GA. Perceived exertion: a note on "history" and methods. Med Sci Sports. 1973;5(2):90-3.
34. Jensen MP, Ward LC, Thorn BE, Ehde DM, Day MA. Measuring the cognitions, emotions, and motivation associated with avoidance behaviors in the context of pain: preliminary development of the negative responsivity to pain scales. Clin J Pain. 2017;33(4):325-34.

35. Bohlmeijer E, ten Klooster PM, Fledderus M, Veehof M, Baer R. Psychometric properties of the five facet mindfulness questionnaire in depressed adults and development of a short form. Assessment. 2011;18(3):308-20.

36. Freund W, Weber F, Billich C, Birklein F, Breimhorst M, Schuetz UH. Ultra-marathon runners are different: investigations into pain tolerance and personality traits of participants of the TransEurope FootRace 2009. Pain Pract. 2013;13(7): 524-32.

37. Ord P, Gijsbers K. Pain thresholds and tolerances of competitive rowers and their use of spontaneous self-generated paincoping strategies. Percept Mot Skills. 2003;97(3 Pt 2): 1219-22.

38. Leźnicka K, Starkowska A, Tomczak M, Cięszczyk P, Białecka M, Ligocka M, et al. Temperament as a modulating factor of pain sensitivity in combat sport athletes. Physiol Behav. 2017;180:131-6.

39. Tajet-Foxell B, Rose FD. Pain and pain tolerance in professional ballet dancers. Br J Sports Med. 1995;29(1):31-4.

40. Jensen MP, Moore MR, Bockow TB, Ehde DM, Engel JM. Psychosocial factors and adjustment to chronic pain in persons with physical disabilities: a systematic review. Arch Phys Med Rehabil. 2011;92(1):146-60. 